\title{
Diversity of Actinomycetes in Nitrogen Fixing Root Nodules of Casuarinaequisetifolia and its Impact on Plant Growth
}

\author{
Gunasekera DTW, Gunawardana D \& Jayasinghearachchi H \\ Department of Botany, University of Sri Jayewardenepura, Gangodawila, Sri Lanka
}

\begin{abstract}
Casuarinaequisetifolia (Kasa) is an actinorhizal plant which is used for rehabilitation of poor and disturbed soil throughout the world. Actinorhizal plants which were colonized by Frankia sp. enhance the soil fertility due to frankial colonization in root nodules. However, few studies were reported on non-frankial colonization and its impact on plant growth and soil fertility. Thus this study was carried out to investigate the unrevealed information on actinomycete consortia residing the nitrogen fixing root nodules of $C$. equisetifolia. The actinomycete were successfully isolated from surface sterilized root nodules by using double layered agar plate technique and also isolates were tested on reinfectivity on Casuarina seedlings. Three different actinomycetes were able to identify as Frankia sp., Micromonospora sp., and novel symbiont as Streptomyces sp. from nitrogen fixing root nodules of Casuarina plants. Further, co-existence of all three isolates were observed in nitrogen free and nitrogen enriched Yeast Mannitol Agar medium (YMA). Frankia sp. promoted the shoot and root growth by $87 \%$ and $55 \%$ respectively with nodulation of C. equisetifolia and whereas Micromonospora sp. promoted the root and shoot growth by $28 \%$ without nodulation. In contrast Streptomyces sp. was able to trigger the lateral root formation of C. equisetifolia which indicate the ability of the microorganism to alter host development system. This study provides novel data on root inhabiting Streptomyces sp. which could play a vital role in enhancing plant growth, exchanging complex signals between plant and microorganisms. These results suggest that the root inhabiting microbial consortium of $C$. equsetifolia would significantly contribute to the development of plant growth.
\end{abstract}

KEYWORDS: . equisetifolia, Streptomyces, Frankia, Micromonospora, soil fertility

Corresponding author: H.Jayasinghearachchi, Email: jayasinghe148@gmail.com 


\section{INTRODUCTION}

C. equisetifolia is one of the most important actinorhizal plants which are used for the rehabilitation of infertile and disturbed soils to improve their stability and fertility. In addition, these plants have been frequently used in agroforestry, coastal protection and natural ecosystems.

Previous studies have reported that these actinorhizal plants mainly have a symbiotic association with Frankia sp.. Further, it has also been reported that Micromonospora sp. colonized in root nodules of $\mathrm{C}$. equisetifolia (Valdes et al., 2005).

Several different non-frankiaactinomycetes were isolated from actinorhizal plants including Coriaria (Trujillo et al., 2005), Discaria (Solans et al., 2011), Alnuselaeagnus (Gtari et al., 2004). These associations have been assumed to play a vital role in both plant growth and soil quality (Echbab et al., 2004).

Solans et al., (2011) showed that Micromonospora sp., Streptomyces sp., Actinoplanes sp., enhanced the plant growth and nodulation frequency of Discariatrinervis.

Further, these actinomycetes produced three different phytohormones: indole-3-acetic acid (IAA), gibberellic acid (GA 3), and zeatin at higher levels than those produced by the symbiotic Frankia strain BCU110501 (Ghodhbane-Gtari and Tisa, 2014).

Little is known about non-frankiaactinomycetes and their associations with C. equisetifolia plants. Some of predicted roles of non-frankia associations are nitrogen fixation, helper bacteria hypothesis and plant hormone production, antagonism model.

Thus, it is essential to study about yet unrevealed information about microbial diversity and their beneficial roles on Casuarina plants.
The present study therefore was undertaken to investigate the microbial diversity in nitrogen fixing root nodules of Casuarina plants. Isolation and identification were based on morphological characteristics and microbial growth on synthetic media. Plant assays were also conducted to investigate the ability of isolates to form nitrogen fixing nodules and their effect on plant growth.

\section{MATERIALS AND METHODS}

\subsection{Collection and maintenance of plant material}

Casuarinaequisetifolia plants with nitrogen fixing root nodule were collected from plantation of National Forest Department, Rediyagama, Hambanthota.

\subsection{Isolation and identification of nodule inhabiting actinomycetes}

\section{i. Surface sterilization of root nodules}

Approximately 10 - 15 active root nodules were collected and carefully washed them with running water. The washed nodules were surface sterilized by following a surface sterilization protocol described elsewhere with some modifications (Gommaet al., 2008). In brief, a mixture of $30 \% \mathrm{H}_{2} \mathrm{O}_{2}+70 \%$ ethanol (1: 1 ratio) was used for surface sterilization.

Each nodule was fragmented into individual lobes and placed them in the mixture of $30 \%$ $\mathrm{H}_{2} \mathrm{O}_{2}+70 \%$ ethanol (1: 1 ratio) for 20 minutes. Then lobes were transferred in to $1 \%$ sodium hypochlorite and kept them for $5 \mathrm{~min}$. They were washed several times with sterile distilled water.

\section{ii. Isolation of actinomycetes}

The BAP medium and Yeast Mannitol Agar (YMA) were used to isolate actinomycetes (Vincent (1970). Double layered agar plate 
technique was used for the isolation of actinomycetes from surfaced-sterilized root nodules (Diem et al., 1982).

Some surface sterilized lobes were directly placed on the bottom layer of the plates and then autoclaved $1.5 \%$ agar at around $45{ }^{\circ} \mathrm{C}$ was poured on it.

In addition, macerated nodule suspension was also streaked on the surface of the bottom layer of the plates and covered it with $1.5 \%$ agar as described above. All the inoculated plates were incubated in a candle jar $28 \pm 1^{\circ} \mathrm{C}$ until microbial growth.

\section{iii. Identification of isolated actinomycetes}

Colonies were observed under light microscope (10x4) (Optika B-350, Japan). Filamentous cells of isolates and chains of spores of one isolate were observed under light microscope after staining them with $1 \%(\mathrm{w} / \mathrm{v})$ lacto phenol cotton blue $(10 \times 40,10 \times 100)$.

\section{iv. Determination of inhibitory growth of the isolates in a co-culture}

The YMA plates were divided in to three equal slots and isolated microorganisms were inoculated on divided slots separately. The plates were incubated at $28 \pm 1{ }^{\circ} \mathrm{C}$. Growth of all isolates together was observed.All the isolated cultures were preserved in $70 \%$ glycerol stocks at $-80{ }^{\circ} \mathrm{C}$ until use.

\subsection{Plant assay}

Collected Casuarina sp.seeds from Hambanthota plantation were surface sterilized with $50 \mathrm{~mL}$ of $5 \%$ sodium hypochlorite (Chlorox) with 2 drops of Tween 80 for $5 \mathrm{~min}$. Surface sterilized seeds were aseptically transferred on to sterile moist cotton wool and kept at $25{ }^{\circ} \mathrm{C}$ under dark condition until germination.
Four third volumes $(3 / 4)$ of fifty milliliter culture tubes filled with quarter strength Hoagland solution were used for the plant assay.

Germinated seeds were first grown in quarter strength N-enriched Hoagland solution for two weeks and then two weeks old seedling were transferred to culture tubes filled with quarter strength $\mathrm{N}$-free- Hoagland solution. Seedlings were inoculated (5\%) separately with exponentially grown cultures of each isolate.

Three replicates were maintained in each treatment and all the treatments were arranged in a completely randomly design (CRD) in a green house. Plants were observed for nodule formation, root growth and lateral root formation. Root and shoot length of $2 \frac{1}{2}$ month old plants were recorded.

\subsection{Statistical analysis}

Statistical analysis was carried out by using Mini Tab 16 software (version 16.2.4.0, minitab Ltd (UK), 2014).

\section{RESULTS}

Isolation and identification of root inhabiting actinomycetes from nitrogen fixing root nodules.

A good growth of three different actinomycetes was observed in double layered agar plates, which were incubated at $28{ }^{\circ} \mathrm{C}$ for $10-15$ days. All isolates were found to be Gram positive and showed filamentous growth. One isolate showed or exhibited starfish shape white colonies on BAP medium (Figure 01: A).

Few isolates were found to develop orangepigmented colonies on YMA plates incubated at $28{ }^{\circ} \mathrm{C}$ after 1 week. Another type of actinomycete was observed in white with aerial mycelia and black pigmentation on YMA plates incubated at $28{ }^{\circ} \mathrm{C}$ after 2-5 days under microaerophilic conditions. 
Further showed a similar characteristic such as heaped, velvety looking colony characteristics to that of genus Streptomyces (Figure 2: A and B). Light microscopy was conducted to identify distinctive morphological characteristics of cells of three isolates when grown in synthetic media. Frankia sp. showed branched and septate hyphae with diazovesicles (Figure 01: B and C).

These diazovesicles were observed in Frankia sp. when it was grown in both solid and liquid medium of BAP, after incubation at $28{ }^{\circ} \mathrm{C}$ for 2 weeks. However, the density of diazovesicles was found to be high when it was grown inNfree liquid medium.

The observed orange color colonies showed branched, septate hyphae without forming any diazovesicles which is similar to that of genus Micromonospora (Figure 01: D).

Mycelia of Streptomyces sp. were found to be more abundant on surface of the YMA plates. Sporulation found to occur on aerial mycelia and chains of spores were produced after incubation under microaerophilic conditions at $28{ }^{\circ} \mathrm{C}$ for 5 days in YMA.

Growth rate of Streptomyces sp. were found to be high when it compared to those of, Frankia sp. and Micromonospora sp. which were grown in liquid medium of YMA incubated at $28{ }^{\circ} \mathrm{C}$ for 5 days.

Dry cell weight of Frankia sp. was found to be low out of three isolates in $\mathrm{N}$-enriched the medium. Frankia sp. showed the lowest growth rate while Streptomyces sp. showed the highest growth rate in Yeast Mannitol broth (Table 01).

Similar growth patterns of isolates were observed in N-free liquid yeast mannitol broth. The ability of the isolate to grow in the presence of different cadmium concentrations (within the range of $0-10 \mathrm{mg} / \mathrm{L}$ ) incorporate yeast mannitol broth was used to differentiate different isolates in terms of their tolerance limits to cadmium.
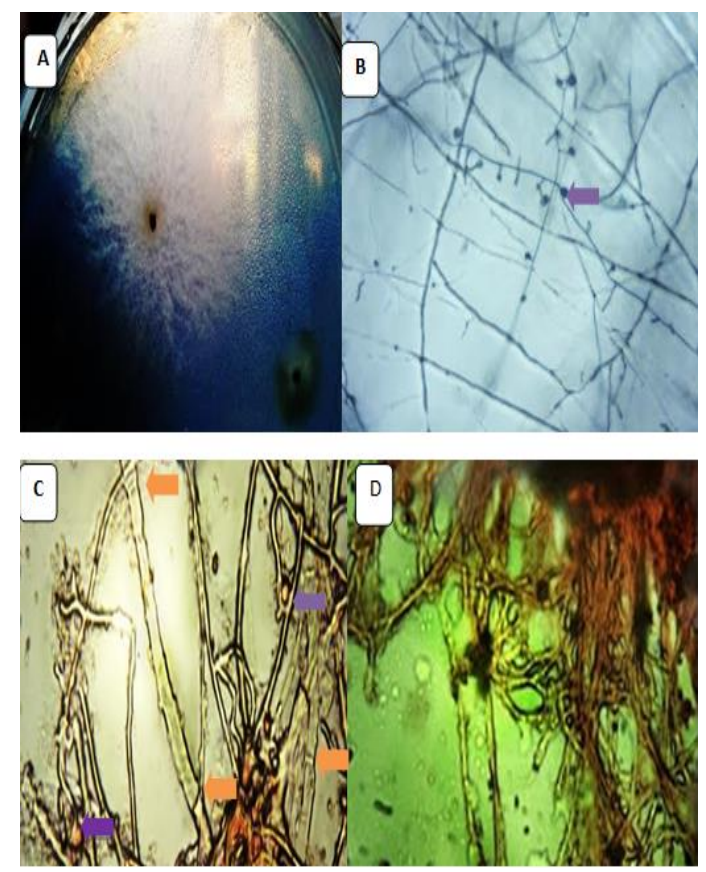

Figure 1. (A-C): Microscopic features of Frankia sp. and Micromonospora sp. isolated from surface sterilized root nodules of $C$. equisetifolia. A) Frankia sp. colony on BAP solid medium. B and C) Frankia diazovesicles on septate mycelia (marked with arrows in orange) and D) diazovesicles of (marked with purple arrow)Micromonospora $\mathrm{sp}$.

Ability of formation of nodules by three isolates was studied in a plant assay. Nodulation was observed only inFrankia sp. treated seedlings. Streptomyces sp. treated seedlings showed increased level of lateral root formation when compared to that of other untreated seedlings.

Plant growth promoting activities of each isolates were determined by measuring the shoot/root length and height of seedlings. The data was shown in the Table 01.In current study, the seedlings which were treated with Frankiasp. showed the highest root $(12.4 \pm 0.80$ $\mathrm{cm})$ and shoot length $(19.3 \pm 1.06 \mathrm{~cm})$ when compared to those of other treated and control seedlings. Data showed that along with different treatments, the plant shoot and root growth increased significantly $(\mathrm{P}<0.005)$. 


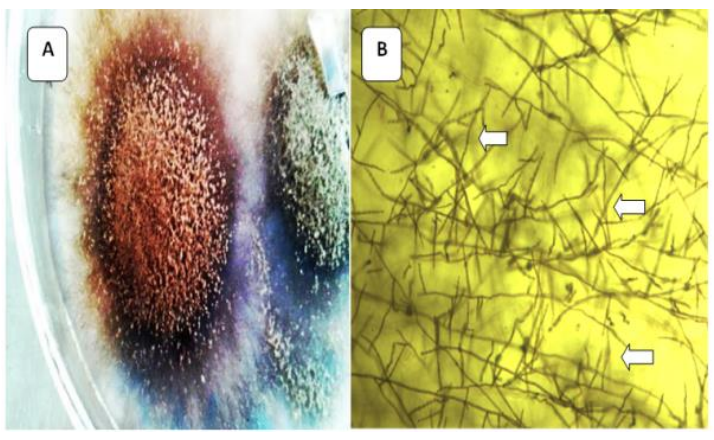

Figure 2.(A-B): Light microscopic view of morphological characteristics of Streptomyces sp. isolated from surface sterilized root nodules of C. equisetifolia.A) Growth of Streptomyces sp. on YMA plate. B) Spore chains of Streptomyces sp. on YMA plate (White arrowsspore chains)

Table 1.Effect of isolated actinomycetes on growth of shoot and roots of Casuarinaequisetifoliaseedlingsgrown in Hoagland's solution

\begin{tabular}{|l|l|l|}
\hline Treatment & $\begin{array}{l}\text { Shoot } \\
\text { length }(\mathbf{c m}) \\
\text { Mean } \pm \text { SE }\end{array}$ & $\begin{array}{l}\text { Root length } \\
(\mathbf{c m}) \\
\text { Mean } \pm \text { SE }\end{array}$ \\
\hline Control & $10.3 \pm 1.02$ & $8.0 \pm 0.96$ \\
\hline Frankia & $19.3 \pm 1.06$ & $12.4 \pm 0.80$ \\
\hline Micromonospora & $13.1 \pm 0.99$ & $10.1 \pm 0.94$ \\
\hline Streptomyces & $10.3 \pm 1.67$ & $9.5 \pm 1.50$ \\
\hline
\end{tabular}

\section{SE- Standard Error}

Seedlings which were treated with Frankia sp. showed highest root and shoot elongation and Streptomyces sp. inoculated seedling showed the triggering effect of lateral root formation (Figure 3:C).

Co-existence activity was observed in triple and dual co-cultures of Frankia sp. with Streptomyces sp., Micromonosporasp. with Streptomyces sp. and Frankia sp., Micromonospora sp. and Streptomyces sp. respectively in YMA media. Inhibition zones were not observed in any of the plates.
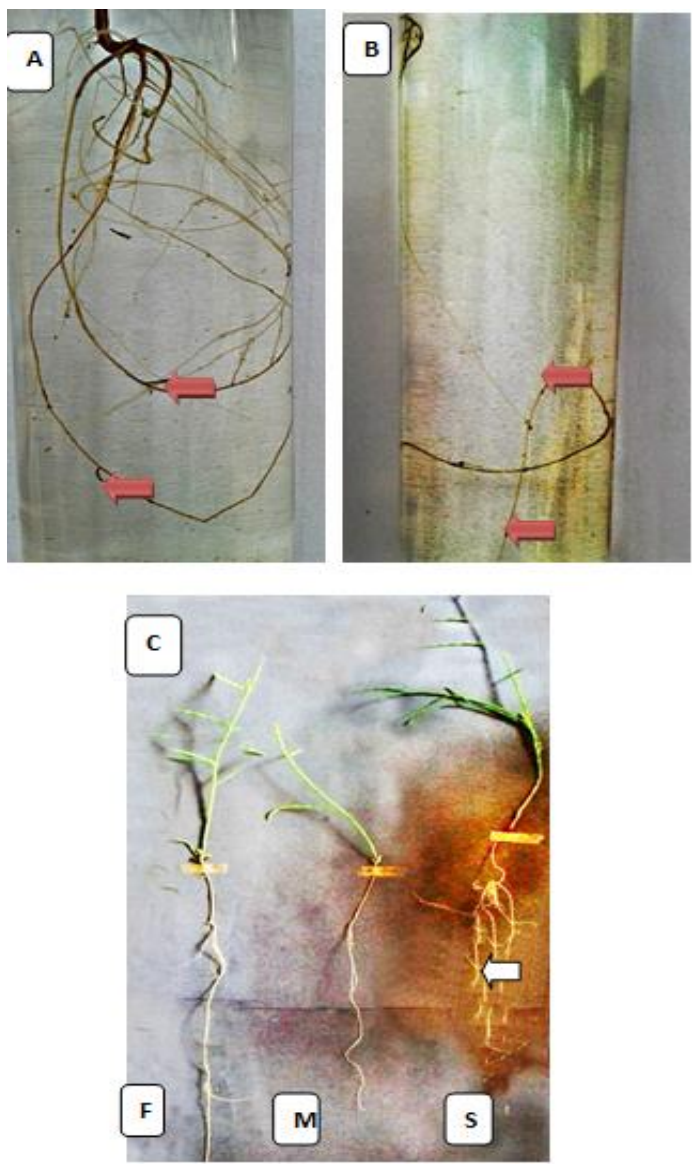

Figure 3. A and B) Nodule primodia of seedling treated with Frankia sp.arrows in red) C) Triggering effect of Streptomyces sp. on Casuarina seedlings (F-Frankia sp., MMicromonospora sp., S- Streptomyces sp.

\section{DISCUSSION}

All three of isolates were Gram positive and showed filamentous growth which is one of major characteristics of Actinomycetales. Frankia sp. showed white star shape colony with septate, branched mycelia with diazovesicles on BAP solid medium plates. The vesicles are the most definitive structure of characterizing the Frankia genus (Benson and Silvester, 1993). Vesicles are normally initiated only in the presence of a nitrogen free medium or during growth on certain nitrogen sources, such as some amino acids, that cannot be degraded to simpler form, ammonia (Zhogzeet 
al., 1986). The vesicles initially develop as terminal swellings on hyphae or on short side branches. In this study considerable amount of vesicles which were formed in short side branches were observed in growth in BAP solid and liquid media.

As reported by Carroet al, (2013) some isolates developed orange colonies which are resembled to the typical characteristic of Micromonosporaceae. Similar results were observed in this study with raised orange colonies on YMA solid and Yeast Mannitol broth. Micromonospora sp. showed branched, septate, hyphae without diazovesicles. Colonies of Streptomyces sp. showed chalky, heaped folded with aerial and substrate mycelia on YMA medium.

The reasonable explanation for the slow growth of Frankia sp. is their generation time has reported as $15 \mathrm{~h}$ or more according to the study Benson and Silvester, (1993). Kawamoto et al., (2014) have shown that Micromonospora sp. has doubling time as 7-8 $\mathrm{h}$ and it was as 4-6 $\mathrm{h}$ for Streptomyces sp. In present study high number of vesicles was observed in nitrogen free media as compared to that of in nitrogen enriched medium. The explanation for this result can be supported with facts of the study carried by Zhogzeet al., (1986), removal of $\mathrm{NH}_{4}{ }^{+}$from the culture medium resulted in vesicle differentiation. The growth rate under atmospheric $\mathrm{N}_{2}$ and medium supplied carbon source going to lengthen it's doubling time for $24 \mathrm{~h}$. It has been reported by Murryet al., (1984) that when nitrogen was withdrawn from medium growth of the mycelium arrested and initiated vesicle differentiation.

Seedlings which were treated with Frankia sp. showed the nodule formation ability out of three isolates. Further, the plants which were treated with Frankia sp. showed the highest shoot and root development. In contrast seedlings, which were treated with Streptomyces sp. showed the increased lateral root formation than that of other plants. This study was first reported on
Streptomyces sp. colonization on nitrogen fixing root nodules of Casuarinaequisetifoliawhere Frankiasp. and Micromonosporasp. are alreadyknown as inhabiting actinomycetes in nitrogen fixing root nodules.

This tripartite symbiosis could be revealing a new path on Casuarina plant growth. Echbabet al., 2004 shown that the $C$. cunninghamiana plant growth promotion according to three hypotheses as an increase in phyto-hormones responsible for root length enhancement, Development of mycorrhizea, synergistic effect on Frankia growth. Diagneet al., 2013 shown that Casuarina plant roots colonized with endo /arbuscular (AMF) and ectomycorrhizzea (ECM) which increase the nutrient absorption capacity via their extensively distributed network of hyphae. Further they have shown that both AMF and ECM increase the plant resistance to diseases by forming external barrier and restrict the entering of the pathogen on to the plant, and alsoconfer resistance to drought condition. Previous studies have reported that inoculation with Frankia increase $\mathrm{N}_{2}$ fixation by $15-80 \%$, ectomycorrhizal colonization by $10-70 \%$ and endomycorrhizal colonization by $10-50 \%$ with EMF. Micromonosporasp. able to synthesize secondary metabolites that able to inhibit the growth of pathogens like Pythium and Phytophthora(Diagneet al., 2013). This emphasize that the importance of these root colonized actinomycetes on the growth and promotion.

The synergistic effect was shown by all two and triple cultures in vivo. Inhibition zones were not observed in co-cultures, even with well-known antibiotic producing bacteria Streptomyces sp.. This result emphasized that there is a synergistic growth or no inhibition within isolated actinomycetes consortia. Similar results have been reported by Solanset al., (2011) that Ochetophilatrinervi plants, an actinorhizal plant inoculated with Frankia sp. showed high growth rate and length when plants were treated with Streptomyces sp. than control. 
It can be concluded from this study that actinomycetes consortia residing the nitrogen fixing root nodules of $C$. equsetifolia able to play a vital role in enhance the plant growth and soil fertility. Further study on effect of different $\mathrm{pH}$ values, water holding capacity, acidity, and salinity on growth of isolated actinomycetes will improve the usage of Casuarina plant in agroforestry.

\section{ACKNOWLEDGEMENT}

This investigation was supported by Academic and non-academic staff of Department of Botany, University of Sri Jayewardenepura and National Forest Department, Hambantotha.

\section{REFERENCES}

BENSON DR\& SIVESTER WB. Biology of Frankia strains, Actinomycetesymbionts of actinorhizal plants. American Society for Microbiology. 1993;57:293-319.

CARRO L\& PUJIC P.Micromonospora is a normal occupant of actinorhizal nodules. Journal of bioscience.2013;38(4):685-693.

DIAGNE N, DIOUF D, SVISTOONOFF S, KANE A, NOBA K, FRANCHE C, BOGUSZ D \& DUPONNOIS R. Casuarina in Africa: Distribution, role and importance of arbuscularmycorrhizal, ectomycorrhizal fungi and Frankia on plant development. J. of Environmental Mgt. 2013: 128:204-209.

DIEM HG, GAUTHIER D\& DOMMERGUES YR. Isolation of Frankia from nodules of Casuarinaequisetifolia. Can. J. Microbiology. 1982;28:526-530.

ECHBAB H, PRIN Y, DUCOUSSO M, NOURISSIER-MOUNTOU S, LAHLOU H\& ARAHO M.Helper bacteria associated with Casuarinacunninghamiana-Frankia symbiosis: selection of isolates for their effects on plant growth in axenic conditions,
ActaBotanicaGallica:

Botany

Letters. 2004;151:429-440.

GTARI M, BRUSETTI L \& SKANDER G.Isolation of Elaeagnus-compatible Frankiafrom soils collected in Tunisia. FEMS Microbiology Letters. 2004; 234(2):349-355.

GHODHBANE-GTARI F \& TISA LS.Ecology and Physiology of Non- FrankiaActinobacteria from Actinorhizal Plants. Plasticity in PlantGrowth-Promoting and Phytopathogenic Bacteria. 2004;p,27-37.

MURRY MA, FONTAINE MS \& TORREY JG. Growth kinetics and nitrogenase induction in Frankia sp. HFPArI 3 grown in batch culture. Development in plant and soil sciences. 1984;12:61-78.

SOLANS M, VOBIS G, CASSSAN F, LUMA $\mathrm{V} \&$ WALL L. Production of phytohormones by root-associated saprophytic actinomycetes isolated from the actinorhizal plant Ochetophilatrinervis. World Journal of Microbial Biotechnology. 2011;27:2195-2202.

TRUJILLO ME, KROPPENSTEDT RM, SUHUMANN P, CARRO L \& EUSTOQUIO M. Micromonosporacoriariae sp. nov., isolated from root nodules of Coriariamyrtifolia. International Journal of Systematic and Evolutionary Microbiology. 2005;56: 23812385.

VALDES M, PEREZ N, SANTOS EP, MELLADO J, CABRIALS J, NORMAND P\& HIRCH AM. Non-Frankiaactinomycetes isolated from surface sterilized roots of Casuarinaequisetifolia. Applied and Environmental Microbiology.2005;71:460-466.

VINCENT JM. The cultivation, isolation and maintenanceof rhizobia: in manual for the practical study of root nodules bacteria. Oxford scientific. 1970;pp1-13. 
ZHONGZE Z, MURRY MA \& TORREY JG. Culture conditions influencing growth and nitrogen fixation in Frankiasp.HFPCcI3 isolated from Casuarina.Development in plant and soil sciences. 1986;91:3-15. 